\title{
Glargine as a Basal Insulin Supplement in Recovering Critically Ill Patients - An In Silico Study
}

\author{
Normy N. Razak* Jessica Lin ${ }^{* *}$ J. Geoffrey Chase* \\ Geoffrey M. Shaw ${ }^{* * *}$ Christopher G. Pretty* \\ Aaron Le Compte* Fatanah M. Suhaimi* Ummu Jamaludin* \\ * Center for Bioengineering, University of Canterbury, New Zealand \\ ** Department of Medicine, University of Otago Christchurch, New \\ Zealand \\ *** Department of Intensive Care Medicine, Christchurch Hospital, \\ New Zealand
}

\begin{abstract}
Tight glycaemic control is now benefiting medical and surgical intensive care patients by reducing complications associated with hyperglycaemia. Once patients leave this intensive care environment, less acute wards do not continue to provide the same level of glycaemic control. Main reason is that these less acute wards do not have the high levels of nursing resources to provide the same level of glycaemic control. Therefore developments in protocols that are less labour intensive are necessary. This study examines the use of insulin glargine for basal supplement in recovering critically ill patients. These patients represent a group who may benefit from such basal support therapy. In silico study results showed the potential in reducing nursing effort with the use of glargine. However, a protocol using only glargine for glucose control did not show to be effective in the simulated patients. This may be an indication that a protocol using only glargine is more suitable after discharge from critical care.
\end{abstract}

Keywords: hyperglycaemia, insulin glargine, tight glycaemic control, SPRINT, critical care

\section{INTRODUCTION}

Stress-induced hyperglycaemia is prevalent in critical care and can occur in patients with no history of diabetes (Capes et al., 2000; Van Den Berghe et al., 2001). Hyperglycaemia worsens outcomes, increasing the risk of severe infection (Bistrian, 2001), myocardial infarction (McCowen et al., 2000) and critical illnesses such as polyneuropathy and multiple organ failure.

A number of studies have investigated the effects on patient outcomes when blood glucose levels are controlled with insulin, and revealed markedly positive results. The most notable is a study by Van Den Berghe et al. (2001) that showed tightly controlled glucose levels averaging $6.1 \mathrm{mmol} / \mathrm{L}$ reduced cardiac intensive care unit (ICU) patient mortality by $18-45 \%$. The use of intravenous (IV) insulin therapy in ICU patients to lower hyperglycaemia, whether or not a patient is a diagnosed diabetic, became the focus of much discussion following this landmark study. Krinsley (2004) showed a 17-29\% reduction in mortality over a wider ICU population with a higher blood glucose average of $7.75 \mathrm{mmol} / \mathrm{L}$. Finally, the SPRINT protocol reduced mortality $36-47 \%$ with a more critically ill cohort (Chase et al., 2008a).

SPRINT or Specialised Relative Insulin Nutrition Tables protocol (Chase et al., 2008a) is a simple wheeled-based system that modulates insulin and nutritional inputs for tight glycaemic control. SPRINT uses the absolute blood glucose level, change in blood glucose level, and current insulin and nutrition administration rates to identify effectively the patient's insulin sensitivity and respond accordingly. The unique wheel-based designed of SPRINT allows all of these metrics to be incorporated into an essentially nurse-automated protocol.

While many ICU patients are benefiting from tight blood glucose control (TGC), moderate to high levels of hyperglycaemia are still tolerated within less acute wards. The use and benefits of insulin protocols within these units have not yet been widely addressed in the literature (Whitehorn, 2007). The management of glycaemic levels in these units remains under the influence of ineffective standard characterized by a tolerance for hyperglycaemia and a reluctance to use insulin intensively.

Based on current evidence from studies in medical and surgical ICUs, it is logical to expect that the maintenance of normoglycaemia within less acute ward patients would limit potential complications associated with elevated blood glucose levels. This assumption is not unreasonable as patients in the ICU and less acute wards share an accelerated catabolic, hyperglycaemic state that also reduces the immune response. Extending tight control to these wards could minimise rebound hyperglycaemia on discharge to the wards (Goldberg et al., 2004) and minimize the development of (new) infections, thus improving overall patient care.

However, to fully implement TGC in less acute wards pose a challenge as these wards do not have the same nursing 
resources compared to ICU, making constant monitoring and titration difficult. Hence, there is a pressing need for insulin delivery protocols that can be successfully implemented with minimal clinical effort and resources.

This study uses an integrated pharmaco-kinetics/dynamics model of insulin glargine, intravenous insulin and glucose from Wong et al. (2008a,b) and Lin et al. (2010) to simulate less intensive TGC protocols using insulin glargine for provision of basal insulin. A retrospective cohort consisting of 10 metabolically stable patients were selected from a cohort of ICU patient receiving TGC with the SPRINT protocol (Chase et al., 2008a). These patients represent a group that may benefit from less intensive protocols that uses insulin glargine as basal supplement. Effective use of insulin glargine, commonly injected once or twice a day, promises a potential to reduce nursing workload, which has added benefits (Chase et al., 2008b). Effective protocols indicated by positive simulation results can then be considered for a clinical pilot study, moving one step closer to providing TGC in the less acute wards.

\section{SYSTEM MODEL}

The pharmacodynamic model used in this study integrates an insulin glargine compartmental model from Wong et al. (2008a,b) and a generic insulin-glucose model, ICING, from Lin et al. (2010). The glargine model has been validated against literature results (Wong et al., 2008a,b) and the model ICING from Lin et al. (2010) has been clinically validated in data from critically ill patients. The integrated model in this study is defined:

\section{Insulin Glargine Compartmental Model}

Precipitate State:

$$
p_{g l a}=\frac{-k_{p r e p, g l a}(t) p_{g l a}(t)}{1+\frac{k_{\text {prep }, \text { gla }}(t)}{r_{\text {dis, max }}} p_{\text {gla }}(t)}+u_{p, g l a}(t)
$$

Hexameric State:

$$
\begin{aligned}
x_{h, g l a}= & -\left(k_{1, g l a}+k_{d}\right) x_{h, g l a}(t) \\
& \frac{-k_{\text {prep }, \text { gla }}(t) p_{\text {gla }}(t)}{1+\frac{k_{\text {prep }, \text { gla }}(t)}{r_{\text {dis, max }}} p_{\text {gla }}(t)}+u_{h, \text { gla }}(t)
\end{aligned}
$$

Dimeric/Monomeric State:

$$
\begin{aligned}
x_{d m}= & -\left(k_{2}+k_{d m}\right) x_{d m}(t)+k_{1, g l a} x_{h, g l a}(t) \\
& +u_{m, g l a}(t)
\end{aligned}
$$

Interstitium:

$$
\dot{x}_{i}=k_{2} x_{d m}(t)-\left(k_{d i}+k_{3}\right) x_{i}(t)
$$

where all the variables in Equation (1)-(4) are defined in Table 1.

\section{Glucose-Insulin Physiology Model}

$$
\begin{aligned}
\dot{G}= & -p_{G} G(t)-S_{I} G(t) \frac{Q(t)}{1+\alpha_{G} Q(t)} \\
& +\frac{P(t)+E G P_{b}-C N S}{V_{G}}
\end{aligned}
$$

Table 1. Nomenclature for the Insulin glargine compartmental model

$x_{h, g l a}(t)$
$p_{g l a}(t)$
$x_{d m}(t)$
$x_{i}(t)$
$r_{d i s, \max }$
$u_{\text {total,gla }}(t)$
$u_{p, g l a}(t)$
$u_{h, g l a}(t)$
$u_{m, g l a}$
$k_{p r e p, g l a}(t)$
$k_{1}$
$k_{1, g l a}$
$k_{2}$
$k_{3}$
$k_{d i}$
$k_{d}$

Mass in glargine hexameric compt.

Mass in glargine precipitate compt.

Mass in dimer monomer compartment

Mass in the interstitium compt.

Max glargine precip. dissolution rate

Insulin glargine input

Glargine precip. state insulin input

Glargine hexamer state insulin input

Glargine dimer/monomer state insulin input

Glargine precipitate dissolution rate

$[\mathrm{mU}]$

$[\mathrm{mU}]$

$[\mathrm{mU}]$

$[\mathrm{mU}]$

$[\mathrm{mU} / \mathrm{min}]$

$[\mathrm{mU} / \mathrm{min}]$

$[\mathrm{mU} / \mathrm{min}]$

$[\mathrm{mU} / \mathrm{min}]$

$[\mathrm{mU} / \mathrm{min}]$

Hexamer dissociation rate

Glargine hexamer dissociation rate

[min-1]

[min-1]

$[\min -1]$

Dimeric/monomeric insulin transport [min-1] rate into interstitium

Interstitium transport rate into plasma

Rate of loss from interstitium

[min-1]

Rate of diffusive loss from hexameric and dimeric/monomeric state compartments

$$
\begin{aligned}
\dot{Q}= & n_{I}(I(t)-Q(t))-n_{C} \frac{Q(t)}{1+\alpha_{G} Q(t)} \\
\dot{I}= & -n_{K} I(t)-\frac{n_{L} I(t)}{1+\alpha_{I} I(t)}-n_{I}(I(t)-Q(t)) \\
& +\frac{u_{e x}(t)}{V_{I}}+\left(1-x_{L}\right) \frac{u_{e n}}{V_{I}} \\
\dot{P} 1= & -d_{1} P 1+D(t) \\
\dot{P} 2= & -\min _{2}\left(d_{2} P 2, P_{\max }\right)+d_{1} P 1 \\
P(t)= & \min \left(d_{2} P 2, P_{\max }\right)+P N(t) \\
u_{e n}(t)= & k_{1} e^{-\frac{I(t)^{k_{2}}}{k_{3}}} \quad \text { when C-peptide data are } \\
& \text { not available }
\end{aligned}
$$

where all the variables in Equations (5)-(11) are defined in Table 2.

\section{METHOD}

The effectiveness of glargine for blood glucose control is assessed in silico. Patient data were selected retrospectively for the simulation study from a cohort of patients who received insulin therapy under the SPRINT protocol during their stay in the Christchurch Hospital ICU (Chase et al., 2008a). SPRINT uses insulin boluses and modulates feed rate hourly to maintain blood glucose levels within a desirable range of $4.0-6.1 \mathrm{mmol} / \mathrm{L}$. It takes into account of a particular patient's apparent insulin sensitivity at any given time to determine the insulin bolus size and feed rate.

The use of glargine is intended for patients who are recovering from their critical illness, and heamo-stability had been regained. The patients selected for simulation are those who exhibit metabolic stability within 30 hour of ICU admission. Metabolic stability in patients in terms of stable blood glucose-insulin response is defined by:

- Hourly insulin boluses $\leq 3 \mathrm{U}$ for at least 12 hours.

- Hourly feed rate of $\geq 60 \%$ of individual patient's goal feed rate. 
Table 2. Nomenclature for ICING model

$\begin{array}{ll}G & \text { Blood glucose level } \\ Q & \text { Interstitial insulin level } \\ I & \text { Plasma insulin level } \\ E G P & \text { Endogenous glucose production } \\ E G P_{b} & \text { Basal endogenous glucose production } \\ C N S & \text { Central nervous system glucose uptake } \\ p_{G} & \text { Insulin independent glucose removal (ex- } \\ & \text { cluding } C N S \text { ) and the suppression of } \\ & \text { EGP from EGP with respect to } G \\ S_{I} & \text { Insulin mediated glucose removal and the } \\ & \text { suppression of } E G P \text { from } E G P_{b} \text { with } \\ & \text { respect to } G \text { and } Q \\ \alpha_{G} & \text { Saturation parameter for insulin medi- } \\ & \text { ated glucose removal } \\ V_{G} & \text { Plasma glucose distribution volume } \\ P(t) & \text { Glucose appearance in plasma from dex- } \\ & \text { trose intake } \\ n_{I} & \text { Plasma-interstitium insulin diffusion rate } \\ n_{C} & \text { receptor-bound insulin degradation } \\ n_{K} & \text { insulin clearance through kidneys } \\ n_{L} & \text { insulin clearance through liver } \\ \alpha_{I} & \text { Saturation parameter for insulin clear- } \\ u_{e x}(t) & \text { ance through liver } \\ u_{e n}(t) & \text { Exogenous insulin } \\ V_{I} & \text { Endogenous insulin } \\ x_{L} & \text { Insulin distribution volume } \\ P 1 & \text { First pass hepatic clearance } \\ P 2 & \text { Glucose level in stomach } \\ d_{1} & \text { Glucose level in gut } \\ d_{2} & \text { Glucose absorption rate from stomach } \\ D(t) & \text { Enteral dextrose intake } \\ P N(t) & \text { Parenteral dextrose intake } \\ P_{m a x} & \text { Maximal glucose flux from gut to plasma } \\ k_{1} & \text { Basal endogenous insulin production } \\ k_{2}, k_{3} & \text { Generic constants for exponential sup- } \\ & \text { pression of } u_{\text {en }} \text { with elevated } I \\ & \end{array}$

intensity. Specifically, nursing effort intensity is measured by the number of intervention required, which includes measuring blood glucose levels, adjusting feed rates, giving SPRINT IV insulin boluses, and giving glargine boluses. The frequencies of blood glucose measurements, changes in feed rates and IV insulin boluses are governed by the SPRINT protocol (Chase et al., 2008a). In particular, blood glucose levels are measured hourly unless 3 hourly measurements have been within 4-6.1 mmol/L. In which case, measurement frequency is changed to 2 hourly until blood glucose levels fail to stay in the $4-6.1 \mathrm{mmol} / \mathrm{L}$ band.

Four different protocols involving the use of glargine are tested to evaluate their potentials for a clinical pilot study. Table 3 lists the full descriptions of simulation protocols that are carried out in this study.

Table 3. Description of protocol simulations.

\section{$\left[\min ^{-1}\right]$ \\ $\left[\min ^{-1}\right]$ \\ $\left[\min ^{-1}\right]$ \\ $[\mathrm{L} / \mathrm{mU}]$ \\ $[\mathrm{mU} / \mathrm{min}]$ \\ $[\mathrm{mU} / \mathrm{min}]$}

[L]

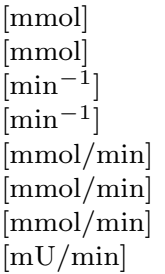

$[\mathrm{mU} / \mathrm{min}]$

Ten patients were chosen from the entire SPRINT cohort (Chase et al., 2008a). Males make up $70 \%$ of the patients selected for the in silico assessments. Median age of these patients is 53.5 [IQR: 44,73] years old. Median APACHE II score is 18 with $\mathrm{IQR}=[12,19]$. The average length of stay is 6.6 days (Range: 5.6-10 days). Mortality is 0 for the selected patients.

Time-varying insulin sensitivity $S_{I}$ was fitted hourly to clinical patient data using Equations (5)-(11) and an integral fitting method (Hann et al., 2005). Constraints are placed on $S_{I}$ to ensure it is within a physiologically valid range. The resulting time-varying $S_{I}$ profiles represent time-varying metabolic status for individual patients. Thus these profiles of $S_{I}$ can act as "virtual patients" for testing different glycaemic control protocols. This "virtual patient" simulation method had been shown to be an accurate way of predicting the effect of different insulin therapies (Chase et al., 2007; Lin et al., 2008).

Because SPRINT operates on the basis of estimating the patient's "apparent" insulin sensitivity (i.e. how much glucose can be removed by the amount of insulin bolus given), the protocol is still applicable when there is a background insulin infusion or similarly, glargine. "Virtual trials" are performed using SPRINT with and without daily doses of glargine. The results from virtual trials are compared to clinical data for the goodness of control, amount of insulin and feed given, and nursing effort

\begin{abstract}
Description Chase et al. (2008a) sulin glargine as a basal insulin replacement therapy sulin glargine where the boluses calculated using SPRINT are reduced by $1 \mathrm{U}$ sulin glargine where the boluses calculated using SPRINT are reduced by $2 \mathrm{U}$ boluses from SPRINT
\end{abstract}

\begin{tabular}{ll}
\hline Protocol & Description \\
\hline Clinical records & Clinical data from Chase et al. (2008a)
\end{tabular}

SPRINT Simulation of the protocol SPRINT

SPRINT+Glargine Simulation of SPRINT protocol with in-

SPRINT-1U+Glargine Simulation of SPRINT protocol with in-

SPRINT-2U+Glargine Simulation of SPRINT protocol with in-

Glargine Only Simulation of daily glargine IV insulin

The dosing frequency of glargine is 24 hours, where the first dose is given at 12 hours after ICU admission. The size of the initial glargine bolus is the sum of SPRINT insulin boluses administered during the previous 12 hours. The following glargine boluses are calculated as being half of the total daily insulin (IV boluses + glargine) from the previous day. Each bolus is capped at $40 \mathrm{U}$ for safety against hypoglycaemia, except when glargine is simulated without the use of SPRINT. In the case where only glargine is used for blood glucose control, a cap of 72 $\mathrm{U} /$ day is used. In addition, clinical records of feed rates are used in the simulations without SPRINT.

\section{RESULTS}

A summary of the results for all 10 patients is shown in Table 4. The blood glucose levels from simulation results of SPRINT are very similar to clinical records, confirming the validity of the virtual trial method. The range in feed for SPRINT is much tighter than clinical records. This can be expected as feed rate is often turned off for patients in real life during various medical procedures. The simulation of SPRINT however does not let the feed rate drop to below $30 \%$ of an individual's goal feed rate.

In the simulations of SPRINT+Glargine, a lot more insulin in total is used even though the amount of IV bolus from SPRINT has decreased. The amount of IV insulin bolus used in SPRINT+Glargine protocol is $15 \%$ less than SPRINT, and 10\% less than clinical records. The control in blood glucose levels (BG) is still good, with a median 75\% [IQR: $69.5-80.4 \%$ ] time in $4.0-6.1 \mathrm{mmol} / \mathrm{L}$ 
Table 4. Protocol result summary. Values are shown in median [IQR].

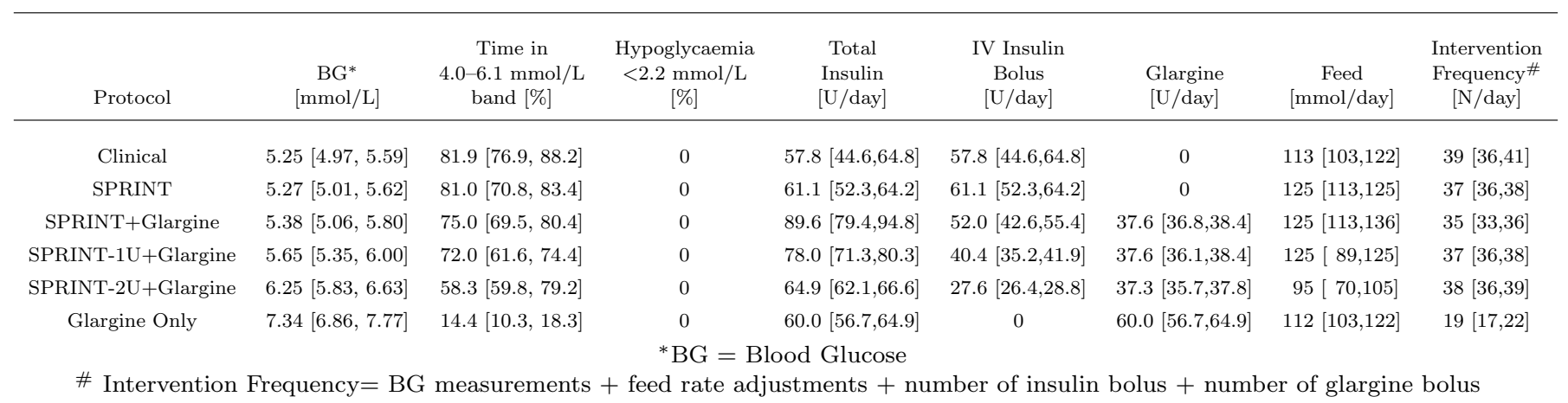

glycaemic band. Feed rate is comparable to SPRINT, however the upper quartile is significantly higher. This is because patients are still given similar amount of insulin towards their end of ICU stay, resulting in patients being able to receive a lot more feed during this time. The most noticeable difference in SPRINT+Glargine compared to SPRINT and clinical records is the reduction in nursing effort, expressed in intervention frequency.

When SPRINT is reduced by 1 and $2 \mathrm{U}$ while glargine is given daily, the tightness of BG is reduced as well as the total amount of insulin used. The amount of feed is also reduced for these two protocols. Because of the loss in tightness of control, intervention frequency is actually not reduced with the use of glargine in these simulations.

The percentage of time spent within desired band reduced to only $14.4 \%$ in the simulation using Glargine only. The daily glargine amount just meet the patient's requirements when comparing to the total amount of insulin used clinically. As glargine is known to have a slow build up, and can take 3 days or more to reach a steady plasma insulin level (Lehmann et al., 2009), the significant decrease in time in band is not a surprise. Finally, all protocols are safe with respect to hypoglycaemia.

Clinical records and simulation results of SPRINT+ Glargine on patient 5122 are shown in Figure 1. The top panel of each subfigure shows the blood glucose levels (BG) through time. BG is well controlled with median and IQR of $5.20[4.90,5.60]$ and $5.14[4.82,5.82]$ for clinical and SPRINT+Glargine respectively. The percentages of time spent within 4.0-6.1 $\mathrm{mmol} / \mathrm{L}$ for the whole duration of patient 5122 's stay are $83.0 \%$ for clinical and $78.3 \%$ for SPRINT+Glargine. There is no occurrence of hypoglycaemia described at $\mathrm{BG}$ below $<2.2 \mathrm{mmol} / \mathrm{L}$ at any period. The median daily feed given in clinical records is $117 \mathrm{~g} /$ day against $126 \mathrm{~g}$ /day from the SPRINT+Glargine protocol. The simulation results for SPRINT is very similar to clinical records in all respects.

For patient 5122 in Figure 1, the frequency and amount of insulin bolus in clinical data are higher than simulated SPRINT+Glargine. Clinical records showed $17 \%$ more IV insulin than SPRINT+Glargine. The frequency of insulin boluses given for patient 5122 is 144 boluses in clinical records against 125 boluses for SPRINT+Glargine (equivalent to 22 and 18 boluses per day). In terms of nursing effort, clinical records showed 262 interventions in total (40/day) whereas SPRINT+Glargine protocol showed 222 interventions (33/day). The higher intervention frequency is mostly attributed to measuring BG. In the simulations of SPRINT+Glargine, BG never dropped below 4 $\mathrm{mmol} / \mathrm{L}$ whereas there was one such instance in the clinical records, as shown in Figure 1. The avoidance of low BG for patient 5122, probably due to less IV insulin boluses given, contributed to most BG being measured at 2 hourly intervals, cutting back on nursing effort. The reduction in the number of IV boluses in SPRINT+Glargine becomes more observable towards the second half of patient 5122's stay. This could be explained by the slow build up of plasma insulin level from glargine (Lehmann et al., 2009).

\section{DISCUSSION}

This study evaluates the safety and efficacy of using insulin glargine to supplement TGC for patients whose insulin requirement for TGC is stable. These patients constitute a group who require a stable, constant "uplift" in their insulin production during their illnesses. The extra requirement may be due to temporary insulin resistance, brought on by excess catecholamines during illness (Bistrian, 2001; McCowen et al., 2000), or temporary impaired endogenous insulin production.

The simulation results from SPRINT are very similar to clinical records. Therefore the virtual patient simulation method is a realistic way to assess different TGC protocols. The simulations of SPRINT+Glargine in this study shows glargine can be used successfully in patients who are insulin resistant but metabolically stable. The results showed reduced nursing effort while still delivering tight control. The blood glucose levels achieved with SPRINT+Glargine are comparable to SPRINT and clinical records. The feed rates are also comparable in the two simulated protocols and clinical records. The amount of insulin used in SPRINT+Glargine is greater than SPRINT and clinical records. This could be due to the slow build up of insulin glargine, which could take 3 days or longer (Lehmann et al., 2009; Heise et al., 2002).

By reducing the amount of bolus in SPRINT by $1 \mathrm{U}$ while still maintaining insulin glargine (SPRINT-1U+Glargine), the results in terms of $\mathrm{BG}$ level are still comparable to clinical records. However, the simulations of SPRINT-2U+Glargine and Glargine Only are not sufficient to provide effective glycaemic management for these patients even though the total insulin used is comparable to clinical data. This could be explained by the slow build up of insulin glargine as mentioned above. The average stay of 6.6 days may not be adequately long for the 

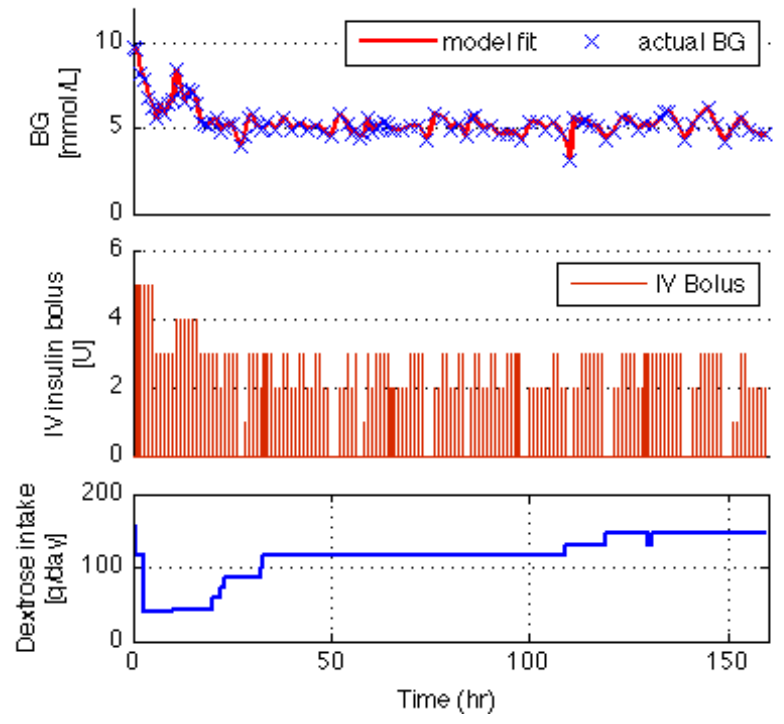

(a) Clinical
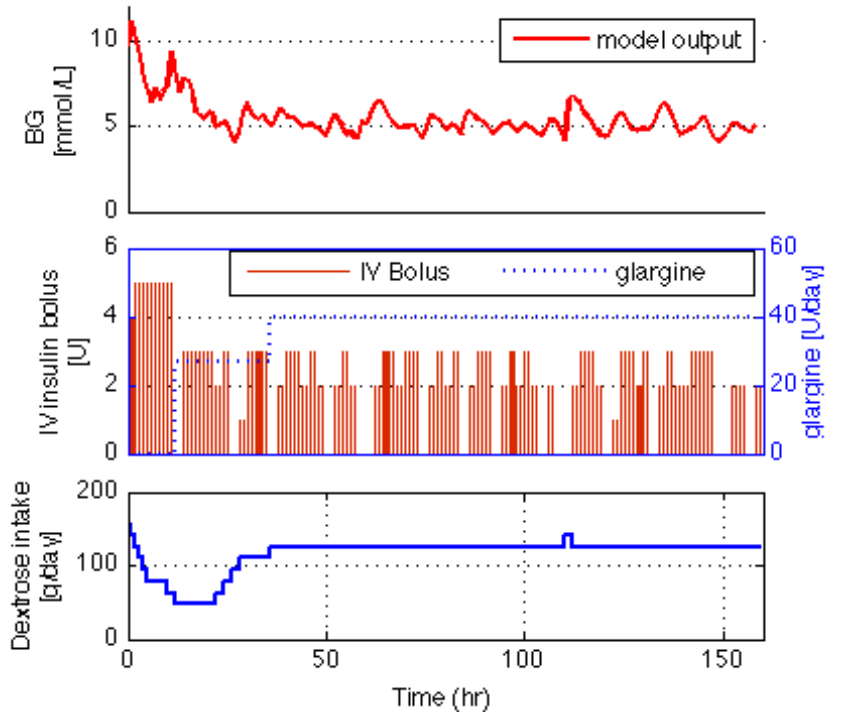

(b) SPRINT+Glargine

Fig. 1. Comparison between clinical and SPRINT+Glargine simulation results on Patient 5122. In the top panel of each subfigure, the solid line (-) illustrates the modeled BG while crosses $(\times)$ are the clinically recorded BG. The second panel of each subfigure shows the insulin bolus $(-)$ and insulin glargine $(\cdots)$. The bottom panel of each subfigure displays the feed.

adaptation of glargine. Even though the patients selected for this study are reasonably stable, critically ill patients in general appear to require more rigorous insulin therapy than using long term insulin supplement such as glargine. This indicates a protocol using glargine only is perhaps only suitable for the less acute wards. However, an early, smooth transition from IV insulin to a combination may help by reducing undesirable variations in blood glucose levels (Egi et al., 2006).

The use of glargine is very conservative in this study, being less than or equal to half of the daily insulin requirement from the previous day. This is to address the course of recovery for patients where they are expected to slowly regain normal insulin sensitivity or basal insulin production.

The protocol SPRINT did not seem to be sensitive to glargine supplementing a patient's insulin requirement. This may be due to the design of SPRINT aiming to achieve a steady state of $3 \mathrm{U}$ insulin and $60 \%$ of feed in patients. In addition, due to the fact that SPRINT is designed in the format of look-up tables, its adjustments in insulin and feed are rather discretized. By reducing SPRINT insulin by $1 \mathrm{U}$ while glargine is given to patients in simulations, the control in blood glucose levels is not compromised. This protocol, with further investigation and revision, may have the potential of being employed in a clinical pilot study. A clinical pilot study will provide valuable information on the practicality and clinical benefit of glargine in stable ICU patient. This will be the first step towards designing glycaemic control protocols for patient in the less acute wards. After all, SPRINT has been proven to provide safe TGC and gained considerable trust in Christchurch Hospital ICU where it is first implemented. A clinical pilot study incorporating SPRINT will be significantly easier to deploy compared to a protocol without it.

Finally, this study only included 10 patients, therefore its results are only a positive "proof-of-concept", and not conclusive. However, the use of glargine is shown to supplement a patient's basal insulin requirement and has the potential to reduce nursing effort. Further reduction in nursing effort without compromising tight control may be possible by revising the blood glucose measurement frequency ( 3 to 4 hourly) while patients are receiving glargine.

\section{CONCLUSION}

This study investigated the use of glargine as basal insulin support in stable, recovering ICU patients. A validated insulin glargine compartmental kinetics model and an insulin-glucose pharmacodynamic model are used to perform simulation of protocols using glargine. Ten metabolically stable patients who received insulin therapy under SPRINT protocol during their stay in Christchurch Hospital ICU were selected for in-silico assessments. Protocols using daily injection of glargine reduced nursing effort provided blood glucose levels are largely maintained within a desirable range. A protocol using glargine only for glucose control did not show to be effective in the patients studied. This may be an indication that a protocol using glargine only is more suitable for the less acute wards. However, an early, smooth transition from IV insulin to a combination may help by reducing undesirable variations in blood glucose levels. Finally, the use of glargine is shown to supplement a patient's basal insulin requirement without the risk of hypoglycaemia. 


\section{REFERENCES}

Bistrian, B. (2001). Hyperglycemia and infection: Which is the chicken and which is the egg? JPEN J Parenter Enteral Nutr, 25(4), 180-181.

Capes, S., Hunt, D., Malmberg, K., and Gerstein, H. (2000). Stress hyperglycaemia and increased risk of death after myocardial infarction in patients with and without diabetes: a systematic overview. Lancet, 355(9206), 773-778.

Chase, J., Shaw, G., Le Compte, A.J., Lonergan, T., Willacy, M., Wong, X., Lin, J., Lotz, T., Lee, D., and Hann, C. (2008a). Implementation and evaluation of the sprint protocol for tight glycaemic control in critically ill patients: a clinical practice change. Crit Care, 12(2), R49. doi:10.1186/cc6868.

Chase, J.G., Shaw, G.M., Lotz, T., Le Compte, A.J., Wong, J., Lin, J., Lonergan, T., Willacy, M., and Hann, C.E. (2007). Model-based insulin and nutrition administration for tight glycaemic control in critical care. Current drug delivery, 4(4), 283-96.

Chase, J.G., Steen, A., Jensen, K., and Shaw, G.M. (2008b). Impact of human factors on clinical protocol performance:a proposed assessment framework and case examples. Journal of Diabetes Science and Technology, 2(3), 409-416.

Egi, M., Bellomo, R., Stachowski, E., French, C., and Hart, G. (2006). Variability of blood glucose concentration and short-term mortality in critically ill patients. Anesthesiology, 105(2), 244-52.

Goldberg, P., Siegel, M., Sherwin, R., Halickman, J., Lee, M., Bailey, V., Lee, S., Dziura, J., and Inzucchi, S. (2004). Implementation of a safe and effective insulin infusion protocol in a medical intensive care unit. Diabetes Care, 27(2), 461-7.

Hann, C.E., Chase, J.G., Lin, J., Lotz, T., Doran, C.V., and Shaw, G.M. (2005). Integral-based parameter identification for long-term dynamic verification of a glucose-insulin system model. Computer Methods and Programs in Biomedicine, 77(3), 259-70. doi: 10.1016/j.cmpb.2004.10.006.

Heise, T., Bott, S., Rave, K., Dressler, A., and Heinemann, R.R.L. (2002). No evidence for accumulation of insulin glargine (lantus): a multiple injection study in patients with type 1 diabetes. Diabet Med, 19(6), 490-5.

Krinsley, J.S. (2004). Effect of an intensive glucose management protocol on the mortality of critically ill adult patients. Mayo Clin Proc, 79(8), 992-1000.

Lehmann, E.D., Tarin, C., Bondia, J., Teufel, E., and Deutsch, T. (2009). Incorporating a generic model of subcutaneous insulin absorption into the aida $\mathrm{v} 4$ diabetes simulator 3 . early plasma insulin determinations. Journal of Diabetes Science and Technology, 3(1), 109201.

Lin, J., Lee, D., Chase, J.G., Shaw, G.M., Le Compte, A.J., Lotz, T., Wong, J., Lonergan, T., and Hann, C.E.C.E. (2008). Stochastic modelling of insulin sensitivity and adaptive glycemic control for critical care. Computer Methods and Programs in Biomedicine, 89(2), 141-52. doi:10.1016/j.cmpb.2007.04.006.

Lin, J., Razak, N.N., Pretty, C., Le Compte, A., Docherty, P., Parente, J.D., Shaw, G.M., Hann, C.E., and Chase, J.G. (2010). A physiological intensive control insulinnutrition-glucose (ICING) model validated in criti- cally ill patients. Computer Methods and Programs in Biomedicine, - in review.

McCowen, K., Friel, C., Sternberg, J., Chan, S., Forse, R., Burke, P., and Bistrian, B. (2000). Hypocaloric total parenteral nutrition: effectiveness in prevention of hyperglycemia and infectious complications-a randomized clinical trial. Crit Care Med, 28(11), 3606-11.

Van Den Berghe, G., Wouters, P., Weekers, F., Verwaest, C., Bruyninckx, F., Schetz, M., Vlasselaers, D., Ferdinande, P., Lauwers, P., and Bouillon, R. (2001). Intensive insulin therapy in the critically ill patients. The New England Journal of Medicine, 345(19), 1359-67.

Whitehorn, L.J. (2007). A review of the use of insulin protocols to maintain normoglycaemia in high dependency patients. Clinical Nursing, 16(1), 16-27.

Wong, J., Chase, J.G., Hann, C.E., Shaw, G.M., Lotz, T.F., Lin, J., and Le Compte, A.J. (2008b). A subcutaneous insulin pharmacokinetic model for computer simulation in a diabetes decision support role: Model structure and parameter identification. Journal of Diabetes Science and Technology, 2(4), 658-671.

Wong, J., Chase, J.G., Hann, C.E., Shaw, G.M., Lotz, T.F., Lin, J., and Le Compte, A.J. (2008a). A subcutaneous insulin pharmacokinetic model for computer simulation in a diabetes decision support role: Validation and simulation. Journal of Diabetes Science and Technology, 2(4), 672-680. 